\title{
PHACTR1 and SLC22A3 gene polymorphisms are associated with reduced coronary artery disease risk in the male Chinese Han population
}

\author{
Qingbin Zhao ${ }^{1}$, Huiyi Wei ${ }^{1}$, Dandan Liu' ${ }^{2}$, Baolan Shi ${ }^{3}$, Lei $\mathrm{Li}^{3}$, Mengdan Yan ${ }^{4}$, Xiyang \\ Zhang $^{5}$, Fengjiao Wang ${ }^{5}$, Yongri Ouyang ${ }^{4}$ \\ ${ }^{1}$ Department of Geratology, the First Affiliated Hospital of Xi'an Jiaotong University, Xi'an, Shaanxi, 710061, China \\ ${ }^{2}$ Department of Endocrinology, the First Affiliated Hospital of Xi'an Jiaotong University, Xi'an, Shaanxi, 710061, China \\ ${ }^{3}$ Inner Mongolia Medical University, Hohhot, Inner Mongolia, 010050, China \\ ${ }^{4}$ School of Life Science, Northwest University, Xi'an, Shaanxi, 710069, China \\ ${ }^{5}$ Xi'an Tiangen Precision Medical Institute, Xi'an, Shaanxi 710075, China
}

Correspondence to: Qingbin Zhao, email: zhaoqingbin8244693@163.com

Keywords: coronary artery disease (CAD), single nucleotide polymorphism (SNP), PHACTR1, SLC22A3, case-control study

Received: September 22, 2016

Accepted: November 12, 2016

Published: November 22, 2016

\section{ABSTRACT}

Previous studies showed that PHACTR1 and SLC22A3 are involved in coronary vascular development and are key determinants of cardiovascular disease risk. We conducted a case-control study to examine the effect of SLC22A3 and PHACTR1 single nucleotide polymorphisms (SNPs) on CAD risk among 376 male CAD patients and 388 male healthy controls from China. Eleven SLC22A3 and PHACTR1 SNPs were selected and genotyped using Sequenom Mass-ARRAY technology. Odds ratios (OR) and 95\% confidence intervals (CIs) were calculated using unconditional logistic regression adjusting for age. The rs9381439 minor allele " $A$ " $(O R=0.72 ; 95 \%$ CI $=0.54-0.96$; $p=0.024)$ in an allelic model was associated with reduced CAD risk, as were the rs2048327 "C/C" (OR = 0.60; 95\% CI: 0.37-0.97; $p=0.036)$ and rs1810126 "T/T" $(O R=0.58 ; 95 \%$ CI: $0.36-0.93 ; p=0.024)$ genotypes. Likewise, the rs9349379 "A/G" genotype in a dominant model $(p=0.041)$, the rs1810126 "T/ $C$ " genotype in additive $(p=0.041)$ and recessive $(p=0.012)$ models, and the rs2048327 "C/T" genotype in a recessive model were associated with decreased CAD risk $(p=0.016)$. These results suggest several PHACTR1 and SLC22A3 polymorphisms are associated with decreased CAD risk in the male Chinese Han population.

\section{INTRODUCTION}

Coronary artery disease (CAD), including myocardial infarction, angina pectoris and arteriosclerosis of the coronary arteries, is a leading cause of illness, disability and death worldwide, particularly in older people [1-3]. In China, moreover, the prevalence of CAD is increasing. The annual mortality rate due to ischemic heart disease is currently 5\% among males and 3.65\% among females [4]. Several studies have identified gender differences in CAD susceptibility that likely account for the higher mortality rate among males $[5,6]$.

CAD is a multifactorial disease, and both acquired and inherited components are implicated in its etiology [7]. While many CAD risk factors can be ameliorated through lifestyle changes, such as diet and exercise, one's genetic make-up and family history of the disease are not modifiable $[8,9]$. Understanding the genetic factors that contribute to the development of CAD continues to be a crucial element for improving methods of effective prevention, early diagnosis and therapeutic strategies.

Among the genetic factors thought to contribute to the risk of $\mathrm{CAD}$ are gene single-nucleotide polymorphisms (SNPs) [10,11]. For example, SNPs in PHACTR1 [12] and SLC22A3 [13] are reported to have a strong association with CAD in Lebanon and Finland, respectively. PHACTRI encodes phosphatase and actin regulator 1 , which can mediate increased oxidation of low-density lipoproteins and is required for vascular development, but may also mediate atherosclerosis in 
humans [14]. SLC22A3 includes 11 exons and 10 introns and encodes organic cation transporter 3 (OCT3), which plays key roles in the inactivation of biogenic amines and the removal of toxic substances [15]. We selected 11 PHACTR1 and SLC22A3 SNPs and assessed their potential to affect CAD risk.

\section{RESULTS}

A total of 376 patients with CAD and 388 healthy controls were enrolled in this case-control study. The demographic characteristics of the study population are shown in Table 1; note the significant difference in the age distribution between the case and control groups $(p<0.001)$.

Table 2 summarizes the minor allelic frequencies (MAFs) of the tested SNPs among the individuals in the case and control groups. All the SNPs in the control group were in Hardy-Weinberg equilibrium (HWE) $(p>0.05)$. The allelic frequencies of SNPs in the control group were similar to those of the HapMap Asian population. Using the $\chi^{2}$ test, we found that rs9381439 located in PHACTR1 was significantly associated with decreased CAD risk $(\mathrm{OR}=0.72 ; 95 \% \mathrm{CI}=0.54-0.96 ; p=0.024)$.

The genotype frequencies of SNPs in the CAD and control groups showed that rs2048327 and rs1810126 were significantly associated with the risk of CAD (Table 3). The rs2048327 "C/C" genotype was associated with a reduced risk of $\mathrm{CAD}(\mathrm{OR}=0.60 ; 95 \% \mathrm{CI}$ : 0.37-0.97; $p=0.036)$, as was the $\operatorname{rs} 1810126$ " $\mathrm{T} / \mathrm{T}$ " genotype $(\mathrm{OR}=0.58$; $95 \% \mathrm{CI}: 0.36-0.93 ; p=0.024)$.

The minor allele of each SNP was assumed to be a risk allele compared to the wild-type allele. Three models - additive, dominant and recessive models - were applied to analyze the association between SNPs and CAD after adjusting for age. We found that PHACTR1 rs9349379 was associated with reduced CAD risk in a recessive model $(\mathrm{OR}=0.71 ; 95 \% \mathrm{CI}=0.51-0.99 ; p=0.041)$. In addition, SLC22A3 rs 1810126 was significantly associated with decreased CAD risk in an additive model $(\mathrm{OR}=0.78$; $95 \% \mathrm{CI}=0.62-0.99 ; p=0.041)$ and recessive model $(\mathrm{OR}=0.59 ; 95 \% \mathrm{CI}=0.39-0.89 ; p=0.012)$. SLC $22 \mathrm{A3}$ rs2048327 was also associated with reduced CAD risk in a recessive model $(\mathrm{OR}=0.59 ; 95 \% \mathrm{CI}=0.39-0.91$; $p=0.016$ ). No other polymorphisms tested were associated with CAD risk in the Chinese Han male population.

\section{DISCUSSION}

In this study we investigated the associations between the 11 SNPs in SLC22A3 and PHACTR1 and CAD risk in the Chinese Han male population. Among these, PHACTR1 rs9381439 and rs9349379 and SLC22A3 rs2048327 and rs1810126 contributed to a decreased risk of CAD in individual SNP analyses. These results suggest that PHACTR1 and $S L C 22 A 3$ polymorphisms may be important determinants of the risk of CAD in the Chinese Han male population.
PHACTR1 is a regulator of protein phosphatase 1 (PP1), an enzyme involved in regulating endothelial production of nitric oxide [16], an important modulator of cardiovascular homeostasis whose activity is elevated in in patients with end stage heart failure $[17,18]$. In a genome-wide association study, PHACTR1 rs9349379 was associated with genetic risk of CAD [19, 20]. Rs9349379 is situated within an intronic region of PHACTR1 and is not in high linkage disequilibrium with any coding SNPs. It may therefore affect transcriptional regulation of PHACTR1 [20]. In our study, we demonstrated that PHACTR1 rs9349379 polymorphism exerts a potentially protective effect against CAD in the Chinese Han male population, which is consistent with an observation made in Lebanese cohort [12]. It could be supposed that the effect of PHACTR1 rs9349379 polymorphism on CAD is not a single-factor process, but consists of complicated elements. We also showed that PHACTR1 rs9381439 is associated with a 0.72 -fold risk of CAD. To the best of our knowledge, this is the first report that this SNP is associated with CAD, though it was a relatively small-scale study, and further studies will be needed to confirm these results.

$S L C 22 A 3$ is located on $6 \mathrm{p} 25.3$ and is a member of the solute carrier super family. It encodes organic cation transporter 3 (OCT3) [21], which modulates intracellular histamine levels by mediating its release and uptake [22]. Histamine is a strong inflammatory mediator that can increase the permeability of vascular endothelial cells and thus increase the deposition of low-density lipoprotein (LDL) within vascular endothelial cells, thereby promoting formation of atherosclerotic plaques [23]. Histamine also induces expression of cytokines, chemokines and adhesion molecules and other inflammatory factors that contribute to atherosclerotic plaque formation [24]. Rs2048327 and rs1810126 reportedly downregulate SLC22A3 transcription and OCT3 protein levels [25]. Individuals carrying these variants may therefore have altered histamine levels, which could greatly affect morbidity. It is also plausible that, by themselves, these polymorphisms have on impact on CAD risk.

Although the present study possesses sufficient power, some limitations should be considered. As we all know, CAD is very heterogeneous disease and has numerous risk factors, including poor nutrition, obesity, hypertension and diabetes. Because the sample size in our study was relatively small, and detailed information was lacking, we could not explore how genetic polymorphisms interact with those factors in CAD. The gene-gene and gene-environment interactions of PHACTR1 and SLC22A3 will need to be evaluated in future studies.

In sum, our study suggests that several PHACTR1 and $S L C 22 A 3$ gene polymorphisms may exert a protective effect against the CAD in the Chinese Han male population. However, the precise function of these polymorphisms and the mechanisms by which expression of these genes affect CAD risk have yet to be determined. 
Table 1: Characteristics of the patients and controls

\begin{tabular}{|c|c|c|c|c|}
\hline Variables & Case & Control & Total & $p$ \\
\hline \multicolumn{5}{|l|}{ Sex } \\
\hline Male & 376 & 388 & 764 & \\
\hline Age, mean \pm SD & $59.60 \pm 11.35$ & $47.50 \pm 10.64$ & & $<0.001^{\mathrm{a}}$ \\
\hline
\end{tabular}

${ }^{a} p$ value was calculated using Welch's $t$ tests.

Table 2: Allele frequencies in patients and controls and odds ratios estimates for CAD

\begin{tabular}{|c|c|c|c|c|c|c|c|c|c|c|}
\hline \multirow{2}{*}{ SNP ID } & \multirow{2}{*}{ Genes } & \multirow{2}{*}{ Band } & \multirow{2}{*}{ Role } & \multirow{2}{*}{ Alleles $\mathbf{A}^{\mathrm{a} / \mathrm{B}}$} & \multirow{2}{*}{ HWE $p$} & \multicolumn{2}{|c|}{ MAF } & \multirow{2}{*}{ ORs } & \multirow{2}{*}{$95 \%$ CI } & \multirow{2}{*}{$p$} \\
\hline & & & & & & Case & Control & & & \\
\hline rs9381439 & PHACTR1 & $6 \mathrm{p} 24.1$ & Intron & $\mathrm{A} / \mathrm{G}$ & 0.852 & 0.274 & 0.291 & 0.72 & $0.54-0.96$ & $0.024^{*}$ \\
\hline rs9349379 & PHACTR1 & $6 \mathrm{p} 24.1$ & Intron & $\mathrm{A} / \mathrm{G}$ & 0.609 & 0.122 & 0.163 & 0.92 & $0.74-1.16$ & 0.498 \\
\hline rs4715166 & PHACTR 1 & $6 \mathrm{p} 24.1$ & Intron & $\mathrm{G} / \mathrm{A}$ & 0.701 & 0.258 & 0.273 & 0.88 & $0.71-1.10$ & 0.259 \\
\hline rs4715167 & PHACTR 1 & $6 \mathrm{p} 24.1$ & Intron & $\mathrm{T} / \mathrm{C}$ & 0.906 & 0.283 & 0.309 & 0.89 & $0.71-1.11$ & 0.289 \\
\hline rs4711939 & PHACTR 1 & $6 \mathrm{p} 24.1$ & Intron & $\mathrm{C} / \mathrm{T}$ & 0.809 & 0.279 & 0.303 & 0.92 & $0.74-1.15$ & 0.462 \\
\hline rs 402219 & $S L C 22 A 3$ & $6 \mathrm{q} 25.3$ & Intron & $\mathrm{G} / \mathrm{A}$ & 0.902 & 0.430 & 0.424 & 1.02 & $0.85-1.29$ & 0.842 \\
\hline rs 376563 & $S L C 22 A 3$ & $6 \mathrm{q} 25.3$ & Intron & $\mathrm{T} / \mathrm{C}$ & 0.278 & 0.384 & 0.373 & 1.05 & $0.85-1.29$ & 0.660 \\
\hline rs2174914 & $S L C 22 A 3$ & $6 \mathrm{q} 25.3$ & Intron & $\mathrm{C} / \mathrm{G}$ & 0.525 & 0.396 & 0.399 & 0.99 & $0.80-1.21$ & 0.906 \\
\hline rs2048327 & $S L C 22 A 3$ & $6 \mathrm{q} 25.3$ & Intron & $\mathrm{C} / \mathrm{T}$ & 0.608 & 0.423 & 0.451 & 0.89 & $0.73-1.09$ & 0.272 \\
\hline rs2457576 & $S L C 22 A 3$ & $6 \mathrm{q} 25.3$ & Intron & $\mathrm{C} / \mathrm{G}$ & 0.397 & 0.399 & 0.404 & 0.98 & $0.80-1.20$ & 0.844 \\
\hline rs1810126 & $S L C 22 A 3$ & $6 \mathrm{q} 25.3$ & 3'UTR & $\mathrm{T} / \mathrm{C}$ & 0.474 & 0.420 & 0.455 & 0.87 & $0.71-1.06$ & 0.172 \\
\hline
\end{tabular}

Abbreviations: ${ }^{a}$ Minor allele; SNP, single-nucleotide polymorphism; HWE, Hardy-Weinberg equilibrium; MAF, minor allelic frequency; OR, odds ratio; $95 \%$ CI: 95\% onfidence interval.

${ }^{*} p<0.05$ indicates statistical significance.

Table 3: Association between SNP genotypes and CAD risk in the male Chinese Han population

\begin{tabular}{|c|c|c|c|c|c|c|c|c|c|c|c|c|c|}
\hline \multirow{2}{*}{ SNPs } & \multicolumn{3}{|c|}{ Case } & \multicolumn{3}{|c|}{ Control } & \multicolumn{7}{|c|}{ Genotype model } \\
\hline & $\mathbf{A A}$ & $\mathbf{A B}$ & BB & $\mathbf{A A}$ & AB & BB & BB-OR & AA-OR & $95 \% \mathrm{CI}$ & $p$ & AB-OR & $95 \% \mathrm{CI}$ & $p$ \\
\hline rs9381439 & 7 & 78 & 291 & 9 & 108 & 270 & $\begin{array}{c}1.00 \\
\text { (reference) }\end{array}$ & 0.72 & $0.21-2.44$ & 0.595 & 0.69 & $0.47-1.01$ & 0.057 \\
\hline rs9349379 & 27 & 140 & 209 & 31 & 149 & 206 & 1.00 & 0.68 & $0.37-1.26$ & 0.219 & 0.71 & $0.50-1.01$ & 0.060 \\
\hline rs4715166 & 32 & 148 & 195 & 36 & 166 & 183 & 1.00 & 0.70 & $0.38-1.28$ & 0.249 & 0.79 & $0.56-1.12$ & 0.187 \\
\hline rs4715167 & 31 & 147 & 197 & 34 & 165 & 185 & 1.00 & 0.75 & $0.40-1.38$ & 0.355 & 0.81 & $0.57-1.14$ & 0.229 \\
\hline rs4711939 & 30 & 146 & 200 & 33 & 158 & 194 & 1.00 & 0.77 & $0.41-1.44$ & 0.419 & 1.18 & $0.73-1.91$ & 0.489 \\
\hline rs402219 & 74 & 175 & 127 & 68 & 190 & 126 & 1.00 & 1.18 & $0.73-1.91$ & 0.489 & 0.86 & $0.59-1.24$ & 0.421 \\
\hline rs376563 & 51 & 187 & 138 & 59 & 171 & 157 & 1.00 & 1.11 & $0.67-1.85$ & 0.680 & 1.43 & $0.99-2.04$ & 0.051 \\
\hline rs2174914 & 57 & 184 & 135 & 65 & 179 & 143 & 1.00 & 1.63 & $0.63-1.69$ & 0.894 & 1.24 & $0.86-1.77$ & 0.246 \\
\hline rs 2048327 & 63 & 192 & 121 & 81 & 186 & 119 & 1.00 & 0.60 & $0.37-0.97$ & $0.036^{*}$ & 1.01 & $0.69-1.46$ & 0.979 \\
\hline rs2457576 & 57 & 186 & 133 & 67 & 177 & 141 & 1.00 & 1.00 & $0.61-1.64$ & 0.989 & 1.27 & $0.89-1.83$ & 0.190 \\
\hline rs1810126 & 62 & 192 & 122 & 84 & 185 & 119 & 1.00 & 0.58 & $0.36-0.93$ & $0.024^{*}$ & 0.98 & $0.67-1.41$ & 0.905 \\
\hline
\end{tabular}

$* p<0.05$ indicates statistical significance;

A/B stands for minor/major alleles. 
Table 4: Logistic regression analysis of the association between SNPs and CAD risk

\begin{tabular}{|c|c|c|c|c|c|c|c|}
\hline \multirow{2}{*}{ SNP } & \multirow{2}{*}{ Minor allele } & \multicolumn{2}{|c|}{ Dominant model } & \multicolumn{2}{|c|}{ Additive model } & \multicolumn{2}{|c|}{ Recessive mode } \\
\hline & & OR $(95 \% \text { CI })^{\mathrm{a}}$ & $p$ & OR $(95 \% \text { CI })^{\mathrm{a}}$ & $p$ & OR $(95 \% C I)^{\mathrm{a}}$ & $p$ \\
\hline rs9381439 & A & $0.69(0.48-1.00)$ & 0.052 & $0.73(0.52-1.02)$ & 0.064 & $0.79(0.23-2.67)$ & 0.701 \\
\hline rs9349379 & A & $0.71(0.51-0.99)$ & $0.041^{*}$ & $0.78(0.60-1.00)$ & 0.053 & $0.78(0.43-1.42)$ & 0.415 \\
\hline rs4715166 & $\mathrm{G}$ & $0.78(0.56-1.08)$ & 0.129 & $0.82(0.64-1.06)$ & 0.123 & $0.78(0.44-1.39)$ & 0.398 \\
\hline rs4715167 & $\mathrm{T}$ & $0.79(0.57-1.11)$ & 0.179 & $0.84(0.65-1.19)$ & 0.185 & $0.82(0.45-1.49)$ & 0.518 \\
\hline rs4711939 & $\mathrm{C}$ & $0.82(0.59-1.14)$ & 0.234 & $0.86(0.66-1.11)$ & 0.240 & $0.84(0.46-1.54)$ & 0.567 \\
\hline rs402219 & G & $0.93(0.66-1.32)$ & 0.715 & $1.05(0.83-1.32)$ & 0.685 & $1.29(0.85-1.98)$ & 0.235 \\
\hline rs376563 & $\mathrm{T}$ & $1.35(0.96-1.89)$ & 0.083 & $1,14(0.89-1.44)$ & 0.296 & $0.91(0.57-1.46)$ & 0.709 \\
\hline rs2174914 & $\mathrm{C}$ & $1.18(0.84-1.66)$ & 0.332 & $1.06(0.84-1.34)$ & 0.638 & $0.92(0.59-1.43)$ & 0.701 \\
\hline rs2048327 & $\mathrm{C}$ & $0.87(0.61-1.24)$ & 0.443 & $0.80(0.63-1.01)$ & 0.062 & $0.59(0.39-0.91)$ & $0.016^{*}$ \\
\hline rs2457576 & $\mathrm{C}$ & $1.19(0.85-1.69)$ & 0.298 & $1.05(0.83-1.33)$ & 0.686 & $0.87(0.56-1.36)$ & 0.552 \\
\hline rs1810126 & $\mathrm{T}$ & $0.84(0.59-1.19)$ & 0.343 & $0.78(0.62-0.99)$ & $0.041 *$ & $0.59(0.39-0.89)$ & $0.012 *$ \\
\hline
\end{tabular}

$* p<0.05$ indicates statistical significance;

${ }^{\mathrm{a}} \mathrm{OR}(95 \% \mathrm{CI})$ and $p$-values were adjusted for age

\section{MATERIALS AND METHODS}

\section{Study participants}

All participants in our study were male Han Chinese. Between 2014 and 2016, we recruited a total of 376 patients diagnosed with CAD at the First Affiliated Hospital of Xi'an Jiaotong University and 388 healthy controls. All cases were verified, and the patients were recruited without restriction regarding age or disease stage and were previously in good health. Diagnosis was based on standard coronary angiography, which revealed $\geq 70 \%$ stenosis of the main branch of a coronary artery or aortic stenosis $\geq 50 \%$ ). The patients had no history of cancer, infection, nephropathy or autoimmune disease. Patients with a history of myocardial infarction, stable angina or unstable angina were also classified as CAD subjects. Excluded were subjects with chronic diseases or conditions involving the brain, liver, heart or lung, and patients with more advanced cardiovascular, metabolic or endocrine diseases. Controls were unrelated and were selected randomly. All of the controls were in good health. All were required to have a comprehensive physical examination. None had any kind of cancer, heart disease or endocrinological, metabolic or nutritional disease.

\section{Clinical data and demographic}

After each subject gave written informed consent, they were interviewed by a psychiatrist who collected personal information, which included region of residence, age, education status and family history of cancer. The use of samples was approved by the Human Research Committee of the First Affiliated Hospital of Xi' an Jiaotong University for Approval of Research Involving Human Subjects.

\section{SNP selection and genotyping}

Among the 11 SNPs selected, rs2048327 in SLC22A3 [13] and rs9349379 in PHACTR1 [26] are reportedly associated with CAD; other PHACTRI and $S L C 22 A 3$ gene SNPs were chosen at random $[12,13]$. Minor allele frequencies of all SNPs were $>5 \%$ in the HapMap of the Chinese Han Beijing population. We used a GoldMag-Mini Whole Blood Genomic DNA Purification Kit (GoldMag Co. Ltd. Xi' an City, China) to extract DNA from whole blood. Extracted DNA was quantified using NanoDrop2000. Genotyping was done with the Sequenom MassARRAY RS1000 system using the standard protocol recommended by the manufacturer. The multiplexed SNP MassEXTENDED assay was designed using Sequenom Mass-ARRAY Assay Design 3.0 Software [27]. Data were managed and analyzed using Sequenom Typer 4.0 Software. Genotype was analyzed using a Sequenom MassARRAY RS1000 system and the standard protocol recommended by the manufacturer $[27,28]$.

\section{Statistical analysis}

Statistical analyses were done using Microsoft Excel and SPSS 18.0 (SPSS, Chicago, IL, USA). All $p$ values were two-sided, and $p \leq 0.05$ was considered significant. Control genotype frequencies for each SNP were tested for departure from the HWE using Fisher's exact test. The $\chi^{2}$ test was used to compare the distribution of marker alleles and genotypes in the patients and controls [29]. Unconditional logistic regression analysis, adjusted for age, was used to test odds ratios (ORs) and 95\% confidence intervals (CIs) [30]. Associations between SNPs and risk of CAD were tested in genetic models using SNP Stats software (http://bioinfo.iconcologia.net). Values 
of OR and 95\% CI were calculated as above. Finally, SHEsis software (http://analysis.bio-X.cn/myAnalysis.php) was used to estimate pairwise linkage disequilibrium (LD) and haplotype construction [31].

\section{ACKNOWLEDGMENTS AND FUNDING}

This study was funded by the National Natural Science Foundation of China (NO: 81200098) and Scientific and technological project of Shaanxi Province, China (NO: 2014K11-03-04-06).

\section{CONFLICTS OF INTEREST}

All Authors declare that they have no conflicts of interest.

\section{REFERENCES}

1. Gaziano TA. Cardiovascular disease in the developing world and its cost-effective management. Circulation. 2005; 112:3547-3553.

2. Lopez AD, Mathers CD, Ezzati M, Jamison DT, Murray CJ. Global and regional burden of disease and risk factors, 2001: systematic analysis of population health data. Lancet. 2006; 367:1747-1757.

3. Gaziano TA. Reducing the growing burden of cardiovascular disease in the developing world. Health Affair. 2007; 26:13-24.

4. Wang Q. Molecular genetics of coronary artery disease. Curr Opin Cardiol. 2005; 20:182-188

5. Residori L, Garcia-Lorda P, Flancbaum L, Pi-Sunyer FX, Laferrere B. Prevalence of co-morbidities in obese patients before bariatric surgery: effect of race. Obes Surg. 2003; 13:333-340.

6. Kazemi-Saleh D, Pishgoo B, Farrokhi F, Fotros A, Assari S. Sexual function and psychological status among males and females with ischemic heart disease. J Sex Med. 2008; $5: 2330-2337$.

7. Libby P, Theroux P. Pathophysiology of coronary artery disease. Circulation. 2005; 111:3481-3488.

8. Bruneau BG. The developmental genetics of congenital heart disease. Nature. 2008; 451:943-948.

9. Yusuf S, Hawken S, Ounpuu S, Dans T, Avezum A, Lanas F, McQueen M, Budaj A, Pais P, Varigos J, Lisheng L, Investigators IS. Effect of potentially modifiable risk factors associated with myocardial infarction in 52 countries (the INTERHEART study): case-control study. Lancet. 2004; 364:937-952.

10. Ronald J, Rajagopalan R, Cerrato F, Nord AS, Hatsukami T, Kohler T, Marcovina S, Heagerty P, Jarvik GP. Genetic variation in LPAL2, LPA, and PLG predicts plasma lipoprotein(a) level and carotid artery disease risk. Stroke. 2011; 42:2-9.
11. Boes E, Coassin S, Kollerits B, Heid IM, Kronenberg F. Genetic-epidemiological evidence on genes associated with HDL cholesterol levels: a systematic in-depth review. Exp Gerontol. 2009; 44:136-160.

12. Hager J, Kamatani Y, Cazier JB, Youhanna S, GhassibeSabbagh M, Platt DE, Abchee AB, Romanos J, Khazen G, Othman R, Badro DA, Haber M, Salloum AK, et al. Genome-wide association study in a Lebanese cohort confirms PHACTR1 as a major determinant of coronary artery stenosis. PloS one. 2012; 7:e38663.

13. Sallinen R, Kaunisto MA, Forsblom C, Thomas M, Fagerudd J, Pettersson-Fernholm K, Groop PH, Wessman M, Finnish Diabetic Nephropathy Study G. Association of the SLC22A1, SLC22A2, and SLC22A3 genes encoding organic cation transporters with diabetic nephropathy and hypertension. Ann Med. 2010; 42:296-304.

14. Reschen ME, Gaulton KJ, Lin D, Soilleux EJ, Morris AJ, Smyth SS, O'Callaghan CA. Lipid-induced epigenomic changes in human macrophages identify a coronary artery disease-associated variant that regulates PPAP2B Expression through Altered C/EBP-beta binding. PLoS Genet. 2015; 11:e1005061.

15. Sakata T, Anzai N, Kimura T, Miura D, Fukutomi T, Takeda M, Sakurai H, Endou H. Functional analysis of human organic cation transporter OCT3 (SLC22A3) polymorphisms. J Pharmacol Sci. 2010; 113:263-266.

16. Mount PF, Kemp BE, Power DA. Regulation of endothelial and myocardial NO synthesis by multi-site eNOS phosphorylation. J Mol Cell Cardiol. 2007; 42:271-279.

17. Dudzinski DM, Michel T. Life history of eNOS: partners and pathways. Cardiovasc Res. 2007; 75:247-260.

18. Neumann J, Eschenhagen T, Jones LR, Linck B, Schmitz W, Scholz H, Zimmermann N. Increased expression of cardiac phosphatases in patients with end-stage heart failure. J Mol Cell Cardiol. 1997; 29:265-272.

19. O'Donnell CJ, Kavousi M, Smith AV, Kardia SL, Feitosa MF, Hwang SJ, Sun YV, Province MA, Aspelund T, Dehghan A, Hoffmann U, Bielak LF, Zhang Q, et al. Genome-wide association study for coronary artery calcification with follow-up in myocardial infarction. Circulation. 2011; 124:2855-2864.

20. Coronary Artery Disease Genetics C. A genome-wide association study in Europeans and South Asians identifies five new loci for coronary artery disease. Nat Genet. 2011; 43:339-344.

21. Verhaagh S, Schweifer N, Barlow DP, Zwart R. Cloning of the mouse and human solute carrier 22a3 (Slc22a3/ SLC22A3) identifies a conserved cluster of three organic cation transporters on mouse chromosome 17 and human 6q26-q27. Genomics. 1999; 55:209-218.

22. Schneider E, Machavoine F, Pleau JM, Bertron AF, Thurmond RL, Ohtsu H, Watanabe T, Schinkel AH, Dy M. Organic cation transporter 3 modulates murine basophil functions by controlling intracellular histamine levels. The J Exp Med.. 2005; 202:387-393. 
23. Rozenberg I, Sluka SH, Rohrer L, Hofmann J, Becher B, Akhmedov A, Soliz J, Mocharla P, Boren J, Johansen P, Steffel J, Watanabe T, Luscher TF, et al. Histamine $\mathrm{H} 1$ receptor promotes atherosclerotic lesion formation by increasing vascular permeability for low-density lipoproteins. Arterioscl Throm Vas. 2010; 30:923-930.

24. Kimura S, Wang KY, Tanimoto A, Murata Y, Nakashima Y, Sasaguri Y. Acute inflammatory reactions caused by histamine via monocytes/macrophages chronically participate in the initiation and progression of atherosclerosis. Pathol Int. 2004; 54:465-474.

25. Nies AT, Koepsell H, Winter S, Burk O, Klein K, Kerb R, Zanger UM, Keppler D, Schwab M, Schaeffeler E. Expression of organic cation transporters OCT1 (SLC22A1) and OCT3 (SLC22A3) is affected by genetic factors and cholestasis in human liver. Hepatology. 2009; 50:1227-1240.

26. Reschen ME, Lin D, Chalisey A, Soilleux EJ, O'Callaghan CA. Genetic and environmental risk factors for atherosclerosis regulate transcription of phosphatase and actin regulating gene PHACTR1. Atherosclerosis. 2016; 250:95-105.
27. Gabriel S, Ziaugra L, Tabbaa D. SNP genotyping using the Sequenom MassARRAY iPLEX platform. Current protocols in human genetics / editorial board, Jonathan L Haines [et al]. 2009; Chapter 2:Unit 212.

28. Thomas RK, Baker AC, Debiasi RM, Winckler W, Laframboise T, Lin WM, Wang M, Feng W, Zander T, MacConaill L, Lee JC, Nicoletti R, Hatton C, et al. Highthroughput oncogene mutation profiling in human cancer. Nat Genet. 2007; 39:347-351.

29. Adamec C. [Example of the Use of the Nonparametric Test. Test X2 for Comparison of 2 Independent Examples]. Ceskoslovenske zdravotnictvi. 1964; 12:613-619.

30. Bland JM, Altman DG. Statistics notes. The odds ratio. Bmj. 2000; 320:1468.

31. Shi YY, He L. SHEsis, a powerful software platform for analyses of linkage disequilibrium, haplotype construction, and genetic association at polymorphism loci. Cell Res. 2005; 15:97-98. 